
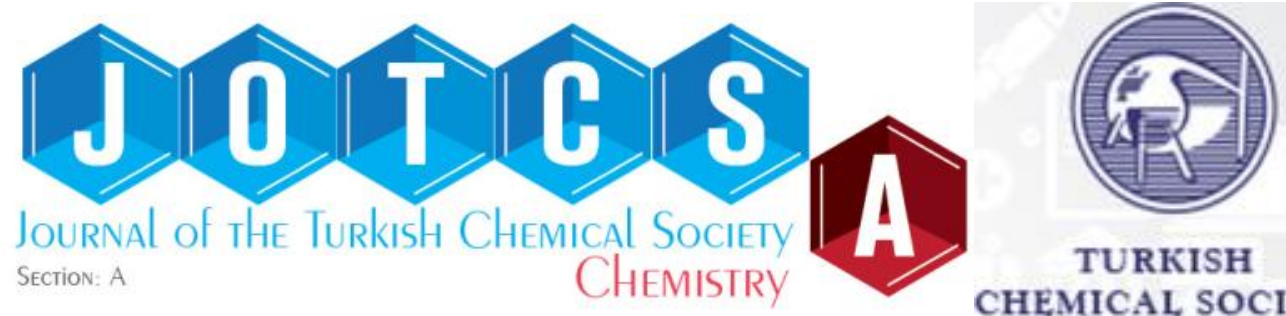

TURKISH

CHEMICAL SOCIETY

\title{
Quantitative Structure-Activity Relationship and Molecular Docking Study of Some Pyrrolones Antimalarial Agents against Plasmodium Falciparum
}

\author{
Zaharaddeen Shehu ${ }^{1 *}$, Adamu Uzairu², Balarabe Sagagi ${ }^{3}$ \\ ${ }^{1}$ Department of Science Laboratory Technology, Jigawa State Polytechnic Dutse, Jigawa State, \\ Nigeria \\ 2 Department of Chemistry, Ahmadu Bello University Zaria, Kaduna State, Nigeria \\ ${ }^{3}$ Department of Chemistry, Kano University of Science and Technology Wudil, Kano State, Nigeria
}

\begin{abstract}
The growing increase in multidrug resistance malaria cases necessitates the need to search for new cost-effective drugs. Quantitative Structure-Activity Relationship (QSAR) and molecular docking studies were performed on a data set of forty-nine Pyrrolones antimalarial agents against Plasmodium falciparum ( $P$. falciparum). Forty-two molecules were used as a training set and seven as the test set. The molecular descriptors were obtained by Density Functional Theory (DFT) using Becke's three-parameter Lee-Yang-Parr hybrid functional (B3LYP) in combination with the 6-31G* basis set. The QSAR model was built using Genetic Function Algorithm (GFA) method. The model with the best statistical significance $\left(N=42, R^{2}\right.$ ext $=0.700, R^{2}=0.933, R^{2} a=0.916$, $\mathrm{Q}^{2} \mathrm{CV}=0.894$, LOF $=0.417$, Minimum experimental error for non-significant LOF $(95 \%)=0.250$ was selected. The docking experiment was carried out using AutoDock Vina of PyRx and Discovery Studio Visualizer. Docking analysis revealed that three of the studied compounds with binding affinity values of $-10.7 \mathrm{kcal} / \mathrm{mol},-10.9 \mathrm{kcal} / \mathrm{mol}$ and $-11.1 \mathrm{kcal} / \mathrm{mol}$ possess higher potency than standard antimalarial drugs with binding affinity values of $-8.8 \mathrm{kcal} / \mathrm{mol},-9.5 \mathrm{kcal} / \mathrm{mol}$ and -9.0 $\mathrm{kcal} / \mathrm{mol}$. It is envisioned that the wealth of information provided by the QSAR and molecular docking results in this study will offer important structural insights for further laboratory experiments in the future design of novel and highly potent antimalarial from the pyrrolones.
\end{abstract}

Keywords: Antimalarial Agents, Density Functional Theory, Genetic Function Algorithm, Discovery Studio Visualizer, Hydrophobic Interaction.

Submitted: October 26, 2017. Accepted: January 15, 2018.

Cite this: Shehu Z, Uzairu A, Sagagi B. Quantitative Structure-Activity Relationship and Molecular Docking Study of Some Pyrrolones Antimalarial Agents against Plasmodium Falciparum. JOTCSA. 2018;5(2):569-84.

DOI: http://dx.doi.org/10.18596/jotcsa.346661.

*Corresponding author. E-mail: zahru2006@yahoo.com, zshehu@jigpoly.edu.ng \& zahru1069@gmail.com. Tel.: +2348061547455. 


\section{INTRODUCTION}

Approximately half of the world's population is at risk of malaria. In 2015, there were roughly 212 million malaria cases and an estimated 429000 malarial deaths. Increased prevention and control measures have led to a $29 \%$ reduction in malaria mortality rates globally since 2010 . Sub-Saharan Africa continues to carry a disproportionately high share of the global malarial burden. In 2015, the region was home to $90 \%$ of malaria cases and $92 \%$ of malaria deaths (1). Among the Plasmodium species responsible for this disease, most commonly encountered and deadliest is Plasmodium falciparum ( $P$. falciparum) (2). In addition to its fatal effects, it has been reported to also have a huge economic impact in countries where it is prevalent. Malaria disease have a huge tendency of spreading to other regions of the world due to increasing globalization and global warming (3). The increasing resistance of $P$. falciparum to clinically used chemotherapeutic agents coupled with the unavailability of vaccines $(3,4,5)$. Though many drugs are abounded for the treatment of this disease, the increasing instances of resistance against antimalarial drugs in recent years are becoming worrisome (6). This underscores the need to discover novel and highly potent drugs in the drug development pipeline to combat the scourge of this disease. In the pursuant of this effort, several molecular drug targets have been identified to develop new drug candidates. Very prominent among these targets are cysteine proteases. This target protein plays an essential role in malaria parasites; therefore, an obvious area of investigation is the inhibition of these enzymes to treat malaria. Studies with cysteine protease inhibitors have revealed its role in hemoglobin hydrolysis. The best characterized Plasmodium cysteine proteases are falcipains, which are papain family enzymes. Falcipain-2 and falcipain-3 are major hemoglobinases of $P$. falciparum (7). In-silico techniques such as QSAR and molecular docking have been proving valuable tools in recent years for rapid discovery of novel drug candidates, e.g. the discovery of o-acetyl-L-serine sulfhydrylase of Entamoeba histolytica inhibitors, acetylcholinesterase inhibitors, and antagonists acetophenazine, fluphenazine and periciazine against human androgen receptor $(8,9,10)$.

QSAR plays a crucial role in drug development as it analyzes the properties of the drug. It is mathematical model that links the structural features of compounds (i.e. molecular descriptors) to their quantity showing specific biological or chemical activity (11). It gives description of how biological activity can vary as a function of molecular descriptors derived from the chemical structure of a set of molecules using regression models. Hence, a model containing those calculated descriptors can be used to predict responses of new compounds (12). The molecular descriptors for the compounds are calculated and used to build QSAR Model (13).

Molecular docking, on the other hand, is a computational method used to determine the binding compatibility of the active site residues to specific groups and to reveal the strength of interaction. 
Shehu, Uzairu and Sagagi. JOTCSA. 2018; 5(2): 569-584.

The results of docking procedure are analyzed by a statistical scoring function which converts interacting energy into numerical values known as the docking score. The 3D pose of the bound ligand can be visualized using different visualizing tools like Pymol, Rasmol, and Discovery Studio, which could help in the inference of the best fit of ligand. Predicting the mode of protein-ligand interaction can assume the active site of the protein molecule and further help in protein annotation. Moreover, molecular docking has major application in drug discovery and designing (14).

The main aim of the present study was to develop various QSAR models using Genetic Function Algorithm (GFA) method for pyrrolones for their antimalarial activities and to relate antimalarial activity to its physicochemical properties. Also to docked falcipain-2 protein (2GHU) with bound ligands (pyrrolone analogues).

\section{MATERIALS AND METHODS}

\section{Dataset of Experimental Falcipain-2 Inhibitors}

A dataset of 49 pyrrolone compounds known as inhibitors of $P$. falciparum was obtained from reference (3) and used in this study. The inhibitory bioactivities of the compounds expressed as half maximal effective concentration $\left(E_{50}\right)$ were converted to the logarithmic scale $\left(p E C_{50}=L_{0 g} E_{50}\right)$ to reduce data dispersion and obtain a more linear response in the process of building the QSAR model. $\mathrm{EC}_{50}$ is the concentration of the drug which induces a response halfway between the baseline and maximum after a specified exposure time (15). It is a commonly used index for assessing the drug's potency. The IUPAC name and biological activities of antimalarial used in this study are presented in Table 1 (See Supplementary Information).

\section{Computational and Statistical Techniques}

The molecular structure of each compound was optimized using the molecular modeling program, Spartan'14 V1.1.0 on Speedstar computer system (4.00 GB RAM, 2.66 GHz processor) on Microsoft windows 10 Ultimate using Density Functional Theory (DFT) level using Becke's three-parameter Lee-Yang-Parr hybrid functional (B3LYP) in combination with the 6-31G* basis set. Our choice of B3LYP/6-311G* basis set is anchored on the fact that it gives a far more accurate result. Optimization of the molecules is necessary to get the lowest energy geometries (most stable geometries). The molecular descriptors of the optimized compounds were calculated using the Spartan'14 V.1.1.0 quantum chemistry package and paDEL descriptor toolkit. The data sets of the molecules were split into the training set (42 molecules) for model development and test set ( 7 molecules) for external validation of the models using Euclidian Distance Based model and the distance between cluster was set at 5. Subsequently, the QSAR models were built by means of Genetic Function Approximation (GFA) techniques embedded in Material Studio, a modeling and 
simulation software using the experimentally obtained biological activities as the dependent variable the computed molecular descriptors as independent variables. Five QSAR models were generated but the best set was selected based on the model with the lowest Lack of Fit (LOF) score, a parameter used for assessing the robustness of GFA derived QSAR model. As a form of quality assurance evaluation of the built QSAR model, the external predictive ability and extrapolation of the best model evaluated using the test set molecules with the aid of Equation 1.

$$
R_{\text {pred }}^{2}=1-\frac{\sum(\text { Pred.Yte }- \text { Act.Yte })^{2}}{\sum(\text { Act.Yte }-Y m)^{2}}
$$

Where $\mathrm{R}^{2}$ pred is termed the predictive $\mathrm{R}^{2}$ of a development model and is an important parameter that is used to test the external predictive ability of a QSAR model, Pred.Yte and Act.Yte indicate predicted and observed activity values of the test set compounds respectively and $Y m$ indicates mean activity value of the training set (17).

\section{Applicabilty Domain of the Model}

Applicability domain $(A D)$ is the physicochemical, structural or biological space, knowledge or information on which the training set of the model has been developed (18). The resulting model can be reliably applicable for only those compounds which are inside this domain since it cannot be applied to the entire universe of anti-P.falciparum compounds. The AD of the model was calculated by the leverage approach (19).

The leverage values of all compounds were calculated and plotted against the standardized residuals (William plot) (20). This method offers a graphical assessment of the leverage values ( $\left.h_{i i}\right)$ as a function of the standardized cross-validated residuals. It is suitable not only for detection of the structurally-influential outliers, but also for determination of the response outliers. The leverage is defined as a compound's distance from the centroid of $X$. Mathematically, the leverage $\left(h_{i i}\right)$ of a given compound in the multidimensional descriptor space, can be calculated as in the following equation:

$$
h_{i i}=x_{i}^{T}\left(X^{T} X\right)^{-1} x_{i}
$$

Where $x_{i}$ the descriptor row matrix of the compound under consideration and $X$ is the multidimensional matrix carrying the structural information (calculated molecular descriptors) for each training set compound.

\section{Molecular docking studies}

In order to have an in-depth knowledge of the nature of the described interaction of inhibitors (3) with the falcipain-2 receptor, molecular docking was performed using AutoDock Vina of PyRx virtual 
screening software. The energy grid calculations and visual analysis of the docking site performed using AutoDock Vina of PyRx and Discovery Studio visualization software, respectively. The crystal structure of falcipain-2 was obtained from protein data bank (PDB CODE: 2GHU). The removal of native ligands present in the protein was confirmed by calculating the root mean square deviation (RMSD) value of the original structure and the structure with ligand deletion $(21,22)$.

All heteroatomic molecules were excluded from the three-dimensional structure of falcipain-2 receptor (Figure 1) and its structure was minimized, protonated and saved in PDBQT file format in all polar residues. Likewise, the 3D structures of the optimized 49 different pyrrolone compounds (Figure 2) were also converted to PDBQT format with the aid of AutoDock 4.2 software. The proteinligand interaction was analyzed using AutoDock Vina of PyRx Virtual Screening software. (23)

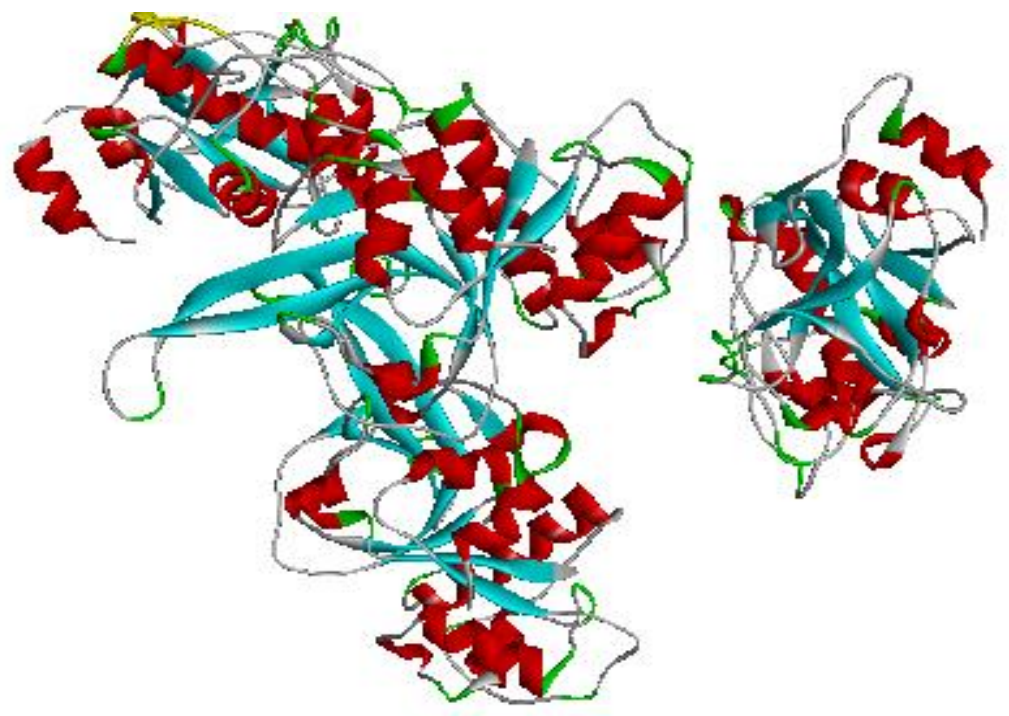

Figure 1: Structure of falcipain-2 (2GHU)

Source: (http://www.pdb.org) (24). 


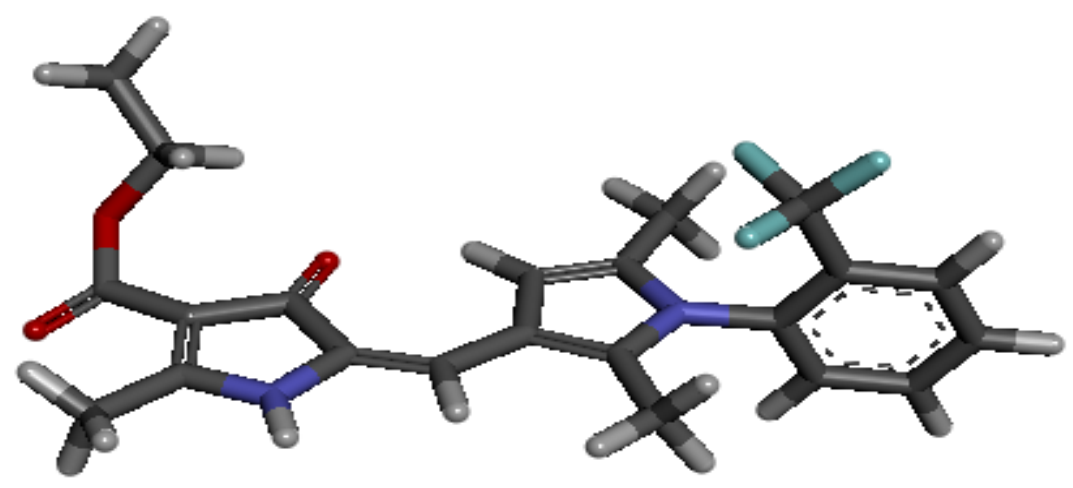

Figure 2: 3D structure of the prepared ligand.

\section{RESULTS AND DISCUSSION}

The five models constructed are detailed as follows:

\section{Model 1}

$p E C_{50}=-3.441($ GATS2m $)+1.934(\operatorname{minsSSN})+1.314($ MDEO -

22) $+3.251($ RDF95u $) \quad-5.776($ RDF130u $)+3.038($ RDF30v $)+$

$2.324(\boldsymbol{R D F 1 5 5 v})-1.739(\boldsymbol{R D F 1 5 s})+7.131$

Model 2

$p E C_{50}$

$$
=\quad-4.943(\text { GATS2m })-1.663(\text { SpMax6Bhs })+1.799(\text { minsss })+1.196(\text { MDEO }-
$$

22) +

$2.707($ RDF95u $)-5.842(\boldsymbol{R D F 1 3 0 u})+2.774(\boldsymbol{R D F 3 0 v})+2.375(\boldsymbol{R D F} 155 v)+7.982$

\section{Model 3}

$p E C_{50}$

$=-4.585($ GATS2m $)+1.959($ maxssSN $)-1.735($ TICO $)+1.261($ MDEO -22$)+$

$3.278($ RDF95u $)-5.290($ RDF130u $)+3.123($ RDF30v $)+2.609($ RDF155v $)+7.276416456$

\section{Model 4}

$p E C_{50}=-4.242($ GATS2m $)+1.783($ maxssSN $)+1.659($ MDEO -22$)+2.908($ RDF95u $)$

- $5.612(R D F 130 u)-1.076(R D F 20 m)+2.999(R D F 30 v)+2.136(R D F 155 v)+7.216$

Model 5

$p E C_{50}$

$=\quad-3.544($ GATS $2 m)-1.983(\boldsymbol{V P})-7+2.459(\boldsymbol{m i n s S S N})+1.218($ MDEO -

22) +

$3.482($ RDF95u $)-6.363($ RDF130u $)+3.007($ RDF30v $)+2.708($ RDF155v $)+7.0614$ 
The validation parameters for the QSAR models are presented in Table 2 and the detailed definition of the descriptors in the model 1 are presented in Table 3. Out of the five models generated, Model 1 shows the best GFA derived QSAR models for inhibitory activity of the studied pyrrolones against P. falciparum. Table 4 gives the comparison of observed $p E C_{50}$ and predicted $p E C_{50}$ of the model 1.

Table 2: Validation Metrics for Models

\begin{tabular}{ccccccc}
\hline Parameter & Model 1 & Model 2 & Model 3 & Model 4 & Model 5 & Recommended \\
\hline $\mathrm{R}^{2}$ & 0.933 & 0.929 & 0.928 & 0.928 & 0.928 & $\geq 0.6$ \\
$\mathrm{R}^{2}{ }_{\mathrm{a}}$ & 0.916 & 0.911 & 0.911 & 0.911 & 0.911 & Very close to $\mathrm{R}^{2}$ \\
$\mathrm{Q}_{\mathrm{CV}}{ }^{2}$ & 0.844 & 0.887 & 0.891 & 0.887 & 0.882 & $<0.5$ \\
$\mathrm{LOF}$ & 0.417 & 0.441 & 0.443 & 0.444 & 0.444 & Very low \\
$\mathrm{R}^{2}$ ext & 0.700 & - & - & - & - & $\geq 0.6$ \\
Error & 0.490 & 0.257 & 0.257 & 0.257 & 0.257 & Very minimal \\
F-value & 56.993 & 53.676 & 53.427 & 53.323 & 53.312 & High \\
\hline
\end{tabular}

Source: Ravinchandran, (2011) (25) 
Shehu, Uzairu and Sagagi. JOTCSA. 2018; 5(2): 569-584.

Table 3: Definition of descriptors in Model 1.

\begin{tabular}{ccr}
\hline S/n & Descriptor & Definition \\
\hline 1 & GATS2m & Geary autocorrelation - lag 2 / weighted by mass \\
2 & MinsssN & Minimum atom-type E-State: $>\mathrm{N}$ - \\
3 & MDEO-22 & Molecular distance edge between all secondary oxygens \\
4 & RDF95u & Radial distribution function - 095 / unweighted \\
5 & RDF130u & Radial distribution function - 130 / unweighted \\
6 & RDF30v & Radial distribution function - 030 / weighted by relative van der Waals volumes \\
7 & RDF155v & Radial distribution function - 155 / weighted by relative van der Waals volumes \\
8 & RDF15s & Radial distribution function - 015 / weighted by relative I-state \\
9 & RDF20m & Radial Distribution Function-020 / weighted by mass \\
10 & MaxsssN & Maximum atom-type E-State: $>\mathrm{N}$ - \\
11 & spMax6Bhs & largest eigenvalue n. 6 of Burden matrix weighted by l-state \\
12 & TICO & Total Information Content index (neighborhood symmetry of 0-order) \\
13 & Vp & Valance path order \\
\hline
\end{tabular}

Source: Descriptor Manual (2012) $(26,27)$ 
Table 4: Comparison of observed $\mathrm{pEC}_{50}$ and predicted $\mathrm{pEC}_{50}$ of Model 1

\begin{tabular}{|c|c|c|c|c|c|}
\hline Cpd & Act. $\mathrm{pEC}_{50}$ & Pred. pEC $_{50}$ & Cpd & Act. pEC 50 & Pred. pEC 50 \\
\hline $1^{a}$ & 7.85 & 7.78 & $26^{a}$ & 6.96 & 7.05 \\
\hline $2^{a}$ & 7.46 & 7.27 & $27^{a}$ & 5.85 & 6.34 \\
\hline $3^{a}$ & 4.92 & 5.00 & $28^{a}$ & 8.39 & 8.23 \\
\hline $4^{a}$ & 5.85 & 5.59 & $29^{b}$ & 9.39 & 9.13 \\
\hline $5^{a}$ & 5.62 & 5.49 & $30^{a}$ & 6.82 & 6.75 \\
\hline $6^{a}$ & 6.72 & 6.39 & $31^{a}$ & 5.92 & 6.51 \\
\hline $7^{a}$ & 5.96 & 5.61 & $32^{b}$ & 7.00 & 8.91 \\
\hline $8^{a}$ & 7.23 & 7.36 & $33^{a}$ & 7.74 & 7.81 \\
\hline $9^{a}$ & 6.42 & 6.77 & $34^{b}$ & 6.00 & 7.11 \\
\hline $10^{a}$ & 6.33 & 6.49 & $35^{a}$ & 7.92 & 7.43 \\
\hline $11^{b}$ & 7.62 & 6.98 & $36^{a}$ & 9.00 & 8.94 \\
\hline $12^{a}$ & 7.64 & 7.62 & $37^{a}$ & 5.82 & 6.21 \\
\hline $13^{a}$ & 8.69 & 8.78 & $38^{a}$ & 8.12 & 7.94 \\
\hline $14^{a}$ & 9.30 & 9.41 & $39^{a}$ & 5.59 & 7.71 \\
\hline $15^{b}$ & 7.62 & 8.18 & $40^{a}$ & 7.21 & 7.24 \\
\hline $16^{a}$ & 6.49 & 7.02 & $41^{a}$ & 7.33 & 7.21 \\
\hline $17^{a}$ & 7.08 & 6.78 & $42^{a}$ & 7.85 & 7.85 \\
\hline $18^{a}$ & 6.89 & 6.97 & $43^{a}$ & 7.68 & 7.64 \\
\hline $19^{b}$ & 7.02 & 6.47 & $44^{a}$ & 6.82 & 7.30 \\
\hline $20^{a}$ & 8.15 & 8.21 & $45^{a}$ & 6.64 & 6.16 \\
\hline $21^{a}$ & 9.00 & 8.91 & $46^{a}$ & 6.60 & 6.86 \\
\hline $22^{b}$ & 5.28 & 6.39 & $47^{a}$ & 6.64 & 6.75 \\
\hline $23^{a}$ & 5.62 & -0.03 & $48^{a}$ & 7.34 & 7.36 \\
\hline $24^{a}$ & 5.46 & 5.61 & $49^{a}$ & 7.11 & 6.99 \\
\hline $25^{a}$ & 7.79 & 7.51 & & & \\
\hline
\end{tabular}

a: training set, b: test set Cpd: compounds, Act: Actual, Pred:Predicted

Figures $3 \mathrm{a}$ and $3 \mathrm{~b}$ give the plot of predicted $\mathrm{pEC}_{50}$ against actual $\mathrm{pEC}_{50}$ of the compounds for the test set and training set, respectively. The William's plot is depicted in Figure 4. 


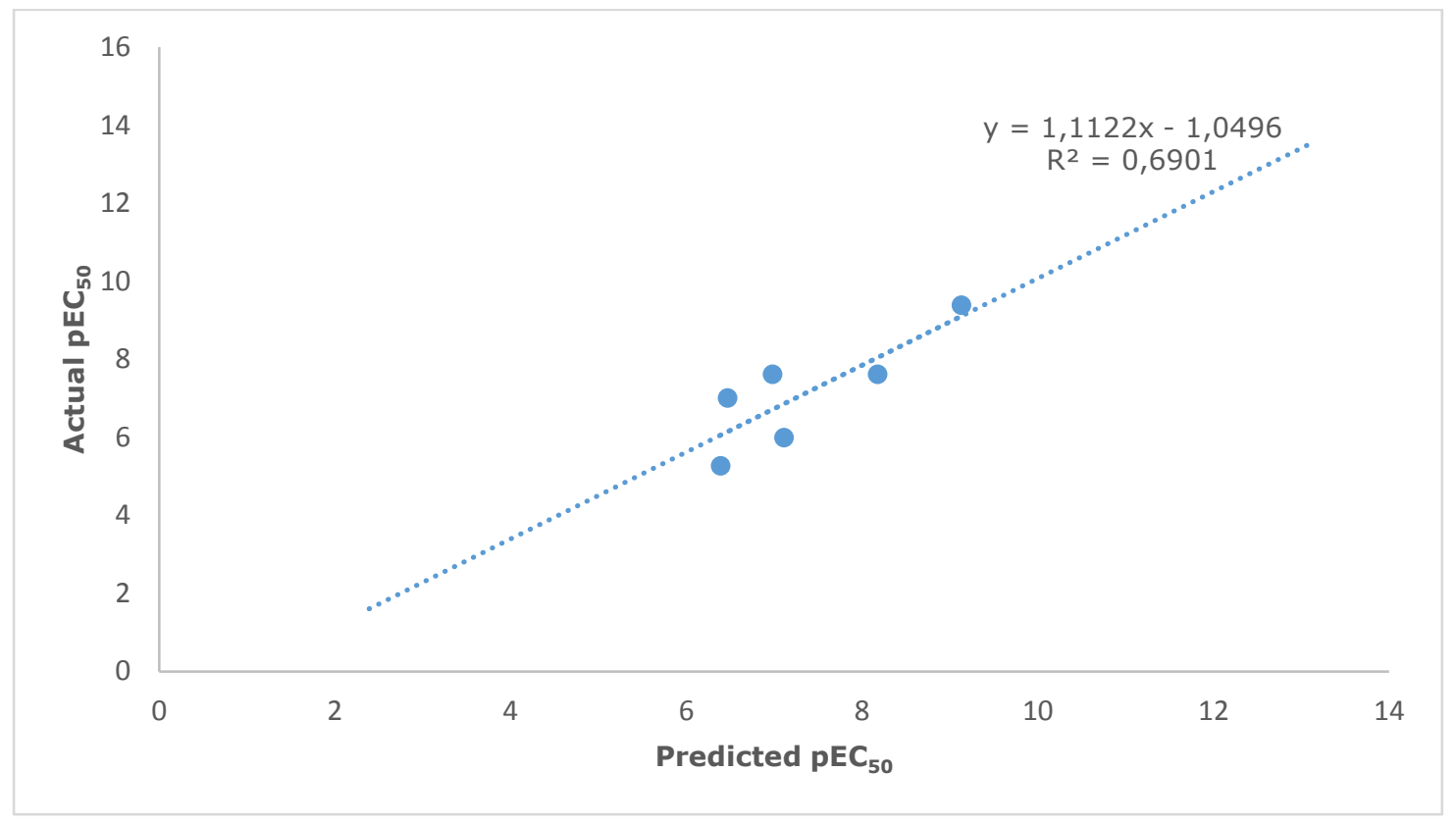

Figure 3a: Plot of predicted versus the observed $\mathrm{pEC}_{50}$ values for the test set compounds.

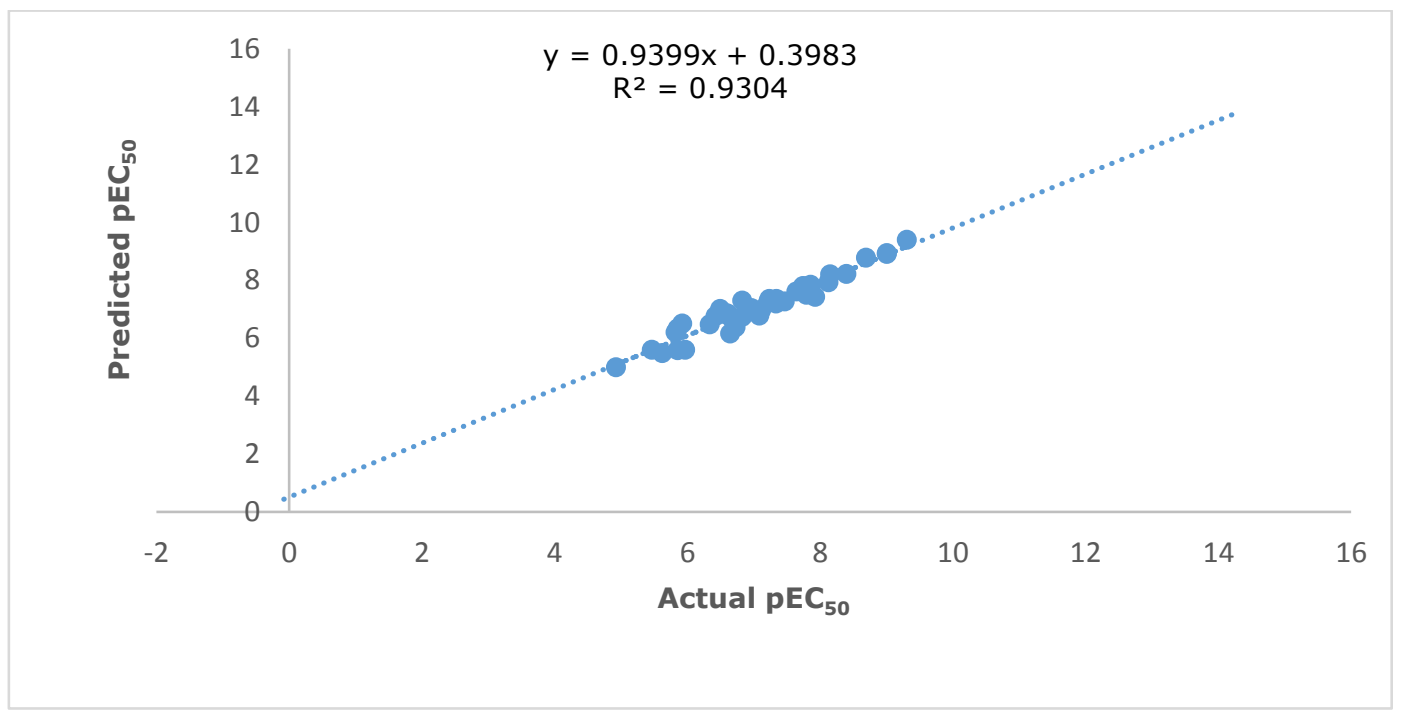

Figure 3b: Plot of predicted versus the observed $\mathrm{pEC}_{50}$ values for the training set compounds 


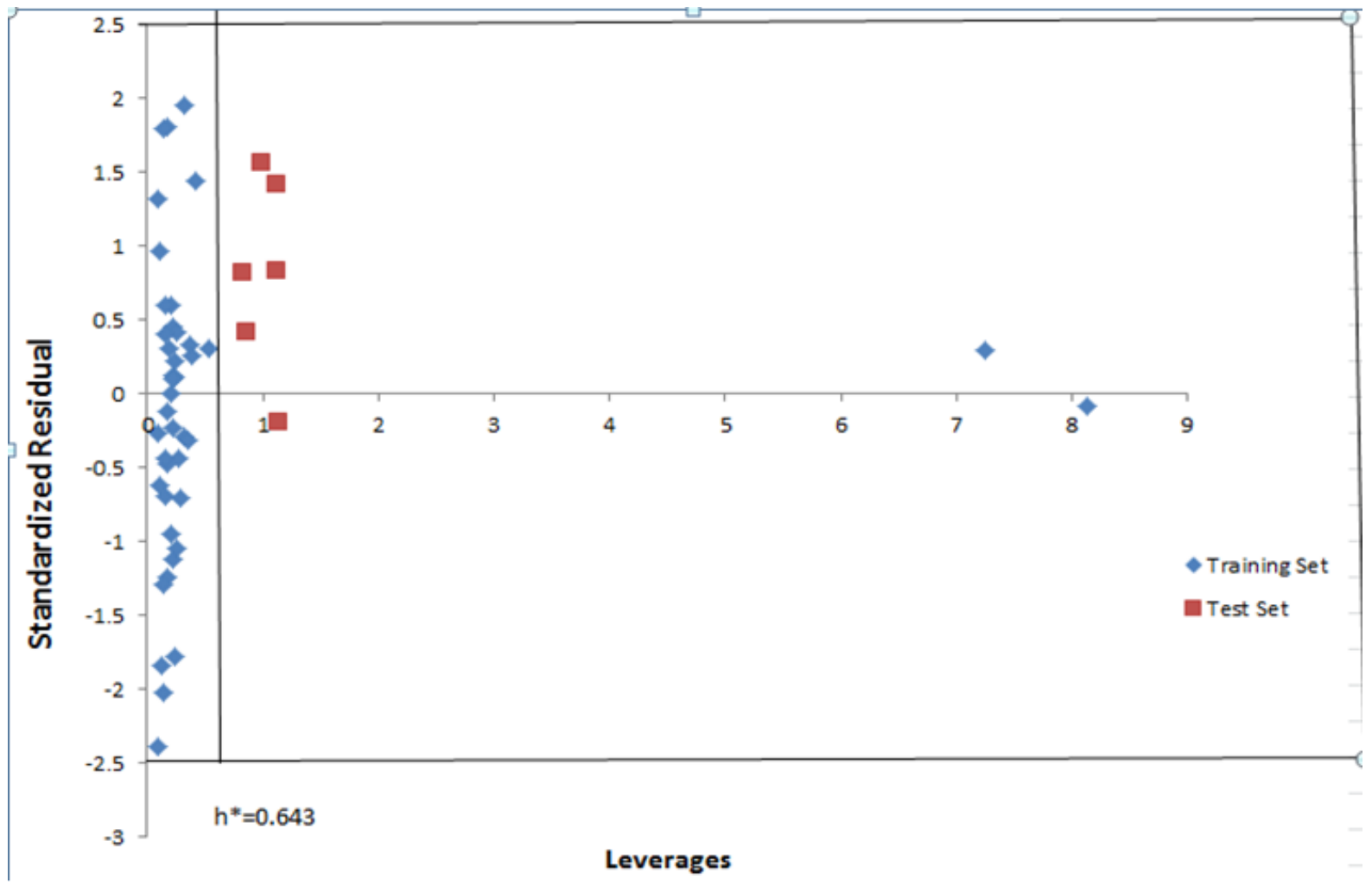

Figure 4. William's plot, the plot of the standardized residuals versus the activity leverage value of model 1.

Table 5 (supplementary files) shows the docking scores, hydrogen bonding length (in Angström) and interacting residues involved in the docking of inhibitors (ligands) at the active site of Falcipain2.

Figures $5 a-c$ show the best three docking results obtained. 
(5a)
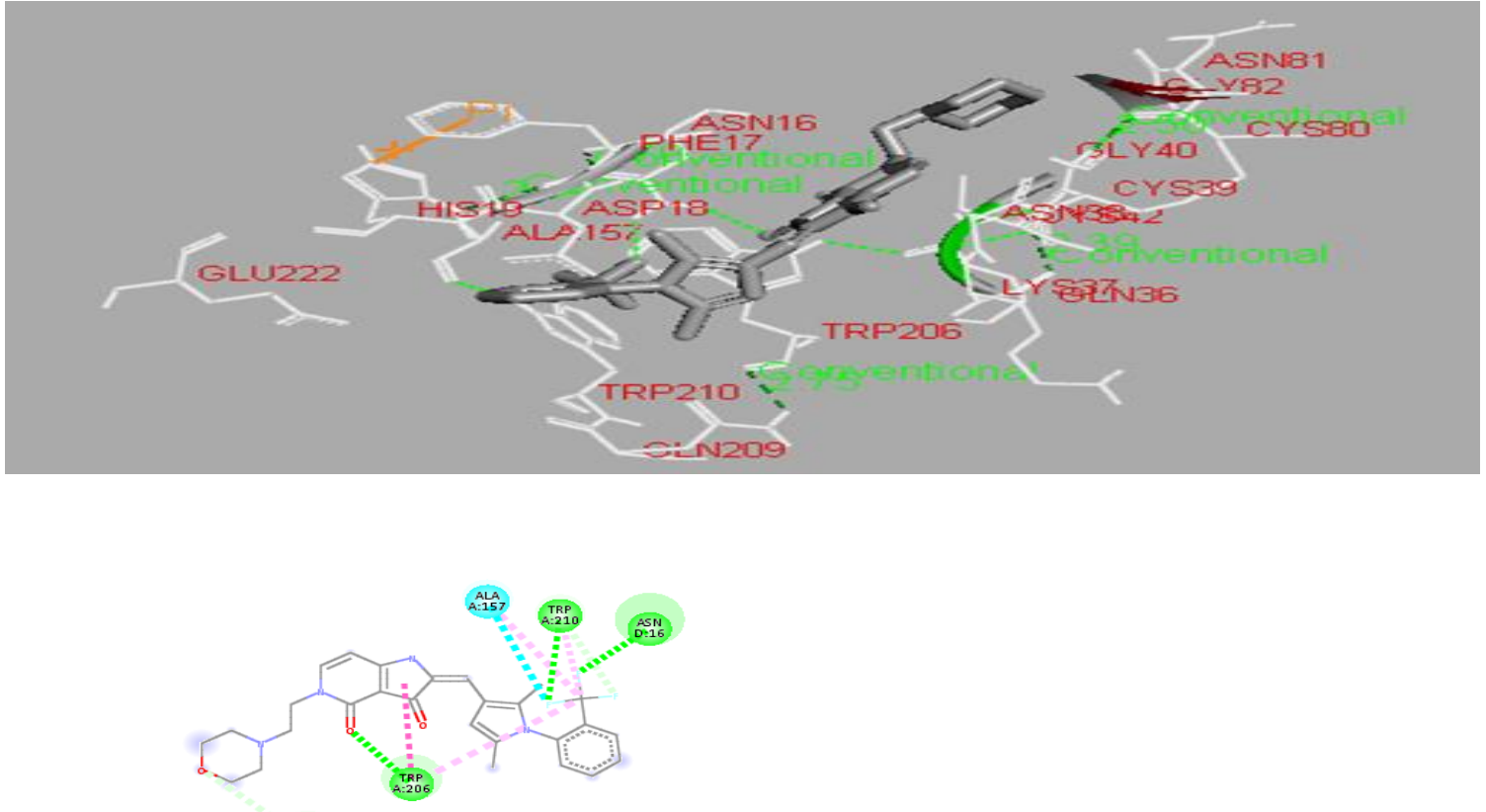

$\underset{\text { AL: }}{\text { GLY }}$

(5b)
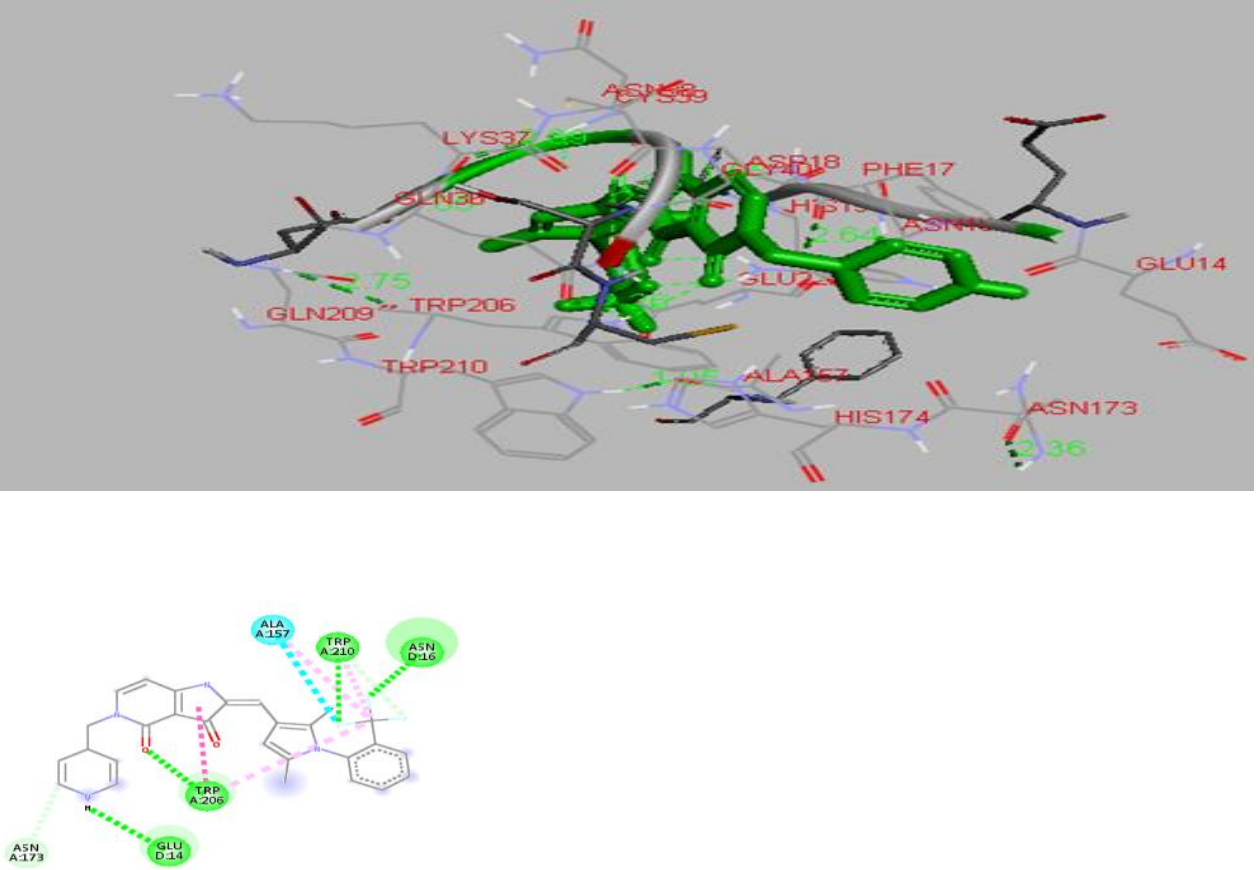

(5c) 

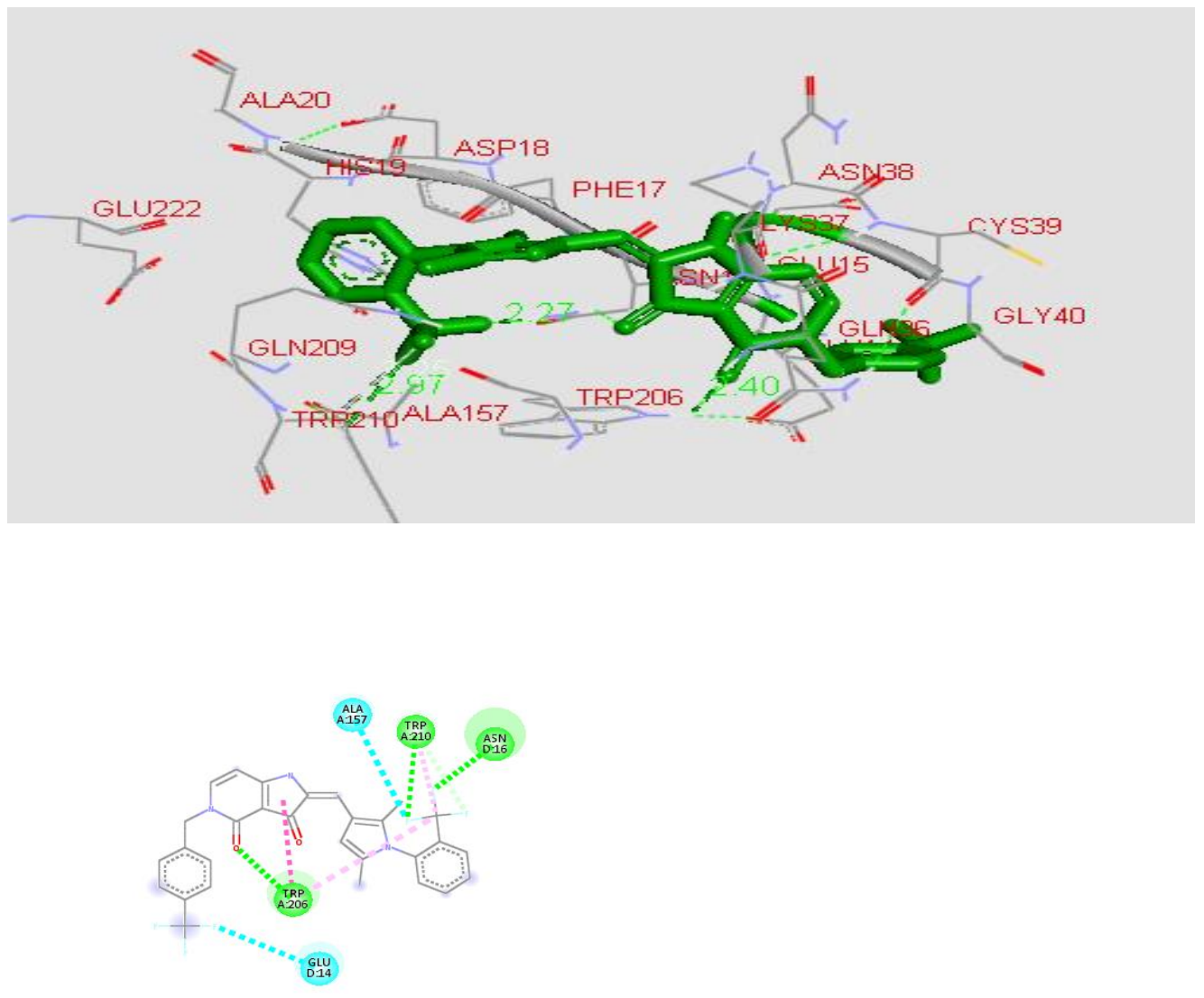

Figure 5: Three-dimensional docked Falcipain-2-Ligands Complex: (5a) Interactions between Falcipain-2 and Ligand 45a, (5b) interactions between Falcipain-2 and Ligand $49^{\mathrm{a}}$ and (5c) interactions between Falcipain-2 and Ligand $48^{\mathrm{a}}$ Green dashed lines represent the $\mathrm{H}$-bond interactions, and red dashed lines represent the hydrophobic interactions.

The statistical parameters of the Genetic Function Algorithm derived QSAR model is in compliance with the standard QSAR validation metrics shown in Table 2. The high predictability of the model is evidenced by the low residual values observed in Table 4 which gives the comparison of observed and predicted anti-P. falciparum activities of the Pyrrolones. Also, the high linearity of the plot of predicted $\mathrm{pEC}_{50}$ against observed $\mathrm{pEC}_{50}$ depicted in Figures $3 \mathrm{a}$ and $3 \mathrm{~b}$ for test set and training set molecules, respectively confirms the robustness of the model.

William's plot (Figure 4) obtained to ascertain the inclusiveness of the studied pyrrolones in model's applicability domain reveals that all the compounds of the training set have leverage values lower than the warning $h_{i}$ value $\left(h_{i}=0.64\right)$ except two compounds with leverage values higher than $h_{i}$. This implies that the models can be successfully applied to this series of pyrrolone antimalarial 
compounds. The two compounds with higher leverage than $h_{i}$ value are most likely to be structural outliers.

Molecular docking studies were carried out between the targets (falcipain-2) and the inhibitors reveal that all the compounds were found to strongly inhibit the growth of $P$. falciparum by completely occupying the active sites of the target protein (falcipain-2). Most of the inhibitors were found to be involved in both hydrophobic interactions and hydrogen bonding with the receptor (falcipain-2).

The best three docking results are shown in Figure 5. Ligand $45^{\mathrm{a}}$ with a binding energy of -10.7 kcal/mol shows hydrophobic interactions Trp210, Trp206, Gln209, GIn36, Lys37, Asn38, Cys39, Cys42, Cys80, Gly82, Asn81, Glu222, Ala157, Asp18, His19, Asn16 and Phe17 with the target in addition to three hydrogen bonding $(2.57 \AA, 2.91 \AA$, and $2.21 \AA)$ with Trp206, Trp210 and Asn16. Ligand $49^{\mathrm{a}}$ with binding score of $-10.9 \mathrm{kcal} / \mathrm{mol}$ forms four hydrogen bonding $(2.26 \AA, 2.92 \AA, 2.22$ $\AA$ and $2.69 \AA$ ) with Trp206, Trp210, Asn16 and Glu14 respectively, and hydrophobic interaction with Trp210, Trp206, Gln209, Gln36, Lys37, Asn38, Cys39, Gly40, Asn16, Asp18, Ala157, His19, Glu222, Phe17, Asn173, Glu14. Similarly, compound $48^{\mathrm{a}}$ with binding score of $-11.1 \mathrm{kcal} / \mathrm{mol}$ forms

hydrogen bonds $(2.40 \AA, 2.97 \AA$, and $2.27 \AA)$ with Trp206, Trp210 and Asn16 respectively, and hydrophobic interactions with Trp210, Trp206, GIn209, Gln36, Lys37, Asn38, Cys39, Gly40, Asn16, Asp18, Ala157, His19, Glu222, Phe17, Glu14, and Glu15.

\section{CONCLUSIONS}

The present study aimed to generate a highly predictive GFAQSAR model capable of revealing the structural requirements for the observed antimalarial inhibitory activities of Pyrrolones against the Plasmodium, P. falciparum. In addition to that, it also investigates the Pyrrolones with best binding affinity to falcipain-2 of the parasite. Falcipain-2 is an essential and validated drug target involved in performing various enzymatic functions such as hemoglobin digestion, erythrocyte invasion, and parasite growth in the host cell. The robustness and applicability of QSAR equation have been established by internal and external validation techniques. The best three docking score is seen in compounds $45^{\mathrm{a}}, 49^{\mathrm{a}}$ and $48^{\mathrm{a}}$, due to their low binding affinity $(-10.7,-10.9$ and $-11.1 \mathrm{kcal} / \mathrm{mol})$ which is higher compared to the standard antimalarial drugs binding affinity value of $(-8.8,-9.5$ and $-9.0 \mathrm{kcal} / \mathrm{mol}$ ) (28). These compounds may be considered for further studies in view of their high potency and binding affinity to the target protein of $P$. falciparum. 
Shehu, Uzairu and Sagagi. JOTCSA. 2018; 5(2): 569-584.

It is envisioned that the wealth of information provided by the QSAR and Molecular docking results in this study will offer important structural insight for further laboratory experiments in the future design of novel and highly potent antimalarial.

In view of the high potency and binding affinity of the compounds to falcipain-2, it is recommended that these compounds be subjected to further laboratory studies. Also, our study advocates the use of combined approaches of QSAR and molecular docking to search for novel potential inhibitors unique to falcipain-2 of $P$. falciparum.

\section{REFERENCES}

1. WHO. 10 facts on malaria [Internet]. World Health Organization; 2016 [cited 2018 Jan 10]. Available from: http://www.who.int/features/factfiles/malaria/en/

2. Newton C. Severe Falciparum Malaria in Children Current Understanding of Pathophysiology and Supportive Treatment. Pharmacology \& Therapeutics. 1998 Jul;79(1):1-53.

3. Kumari M, Chandra S, Tiwari N, Subbarao N. 3D QSAR, pharmacophore and molecular docking studies of known inhibitors and designing of novel inhibitors for M18 aspartyl aminopeptidase of Plasmodium falciparum. BMC Structural Biology [Internet]. 2016 Dec [cited 2018 Jan 13];16(1). Available from: http://bmcstructbiol.biomedcentral.com/articles/10.1186/s12900-016-0063-7

4. Basco LK, Bras JL. In Vitro Activity of Artemisinin Derivatives Against African Isolates and Clones of Plasmodium falciparum. The American Journal of Tropical Medicine and Hygiene. 1993 Sep 1;49(3):301-7.

5. Pandey KC, Dixit R. Structure-Function of Falcipains: Malarial Cysteine Proteases. Journal of Tropical Medicine. 2012;2012:1-11.

6. Bisson WH, Cheltsov AV, Bruey-Sedano N, Lin B, Chen J, Goldberger N, et al. Discovery of antiandrogen activity of nonsteroidal scaffolds of marketed drugs. Proceedings of the National Academy of Sciences. 2007 Jul 17;104(29):11927-32.

7. Mizutani MY, Itai A. Efficient Method for High-Throughput Virtual Screening Based on Flexible Docking: Discovery of Novel Acetylcholinesterase Inhibitors. Journal of Medicinal Chemistry. 2004 Sep;47(20):481828.

8. Nagpal I, Raj I, Subbarao N, Gourinath S. Virtual Screening, Identification and In Vitro Testing of Novel Inhibitors of O-Acetyl-L-Serine Sulfhydrylase of Entamoeba histolytica. Bogyo M, editor. PLoS ONE. 2012 Feb $15 ; 7(2): \mathrm{e} 30305$.

9. Esposito EX, Hopfinger AJ, Madura JD. Methods for Applying the Quantitative Structure-Activity Relationship Paradigm. In: Bajorath J, editor. Chemoinformatics [Internet]. Totowa, NJ: Humana Press; 2004 [cited 2018 Jan 13]. p. 131-213. Available from: http://link.springer.com/10.1385/1-59259-802-1:131

10. Xue L, Bajorath J. Molecular Descriptors in Chemoinformatics, Computational Combinatorial Chemistry, and Virtual Screening. Combinatorial Chemistry \& High Throughput Screening. 2000 Oct 1;3(5):363-72.

11. Barril X, Morley SD. Unveiling the Full Potential of Flexible Receptor Docking Using Multiple Crystallographic Structures. Journal of Medicinal Chemistry. 2005 Jun;48(13):4432-43.

12. Anonymous. EC50 [Internet]. revolvy.com; [cited 2018 Oct 1]. Available from: https://www.revolvy.com/main/index.php?s=EC50\&uid=1575 
13. Anonymous. Stardrop. Optibrium;

14. Veeresamy R, Rajak H, Jain A, Sivadasan S, Varghese C, Agrawal R. Validation of QSAR Models Strategies and Importance. Int J Drug Des Dis. 2011;2(3):511-9.

15. Eriksson L, Jaworska J, Worth A, Cronin M, McDowell R, Gramatica P. Methods for reliability and uncertainty assessment and for applicability evaluations of classification- and regression-based QSARs. Environ Health Perspect. 2003;111(10):1361-75.

16. Gramatica P. Principles of QSAR models validation: internal and external. QSAR \& Combinatorial Science. 2007 May;26(5):694-701.

17. Madeswaran A, Umamaheswari M, Asokkumar K, Sivashanmugam T, Subhadradevi V, Jagannath P. Computational drug discovery of potential phosphodiesterase inhibitors using in silico studies. Asian Pacific Journal of Tropical Disease. 2012 Jan;2:S822-6.

18. Daisy $\mathrm{P}$, Nivedha R, Bakiya RH. In silico drug designing approach for biotin protein Ligase of Mycobacterium tuberculosis. Asian J Pharm Clin Res. 2013;6(I1):103-107.

19. Monika JK, Singh K. Virtual screening using the ligand ZINC database for novel lipoxygenase-3 inhibitors. Bioinformation. 2013;9(11):583.

20. Hogg T, Nagarajan K, Schmidt CL, Hilgenfeld R. Crystal structure of falcipain-2 from Plasmodium falciparum. 2006 Jun 6;

21. Veerasamy R, Rajak H, Jain A, Sivadasan S, Varghese CP, Agrawal RK. Validation of QSAR modelsstrategies and importance. International Journal of Drug Design \& Discovery. 2011;3:511-519.

22. Anonymous. List of molecular descriptors calculated by Dragon [Internet]. Talete; Available from: http://www.talete.mi.it/products/dragon_molecular_descriptor_list.pdf

23. Adejoro I, Waheed S, Adeboye O. Molecular Docking Studies of Lonchocarpus cyanescens Triterpenoids as Inhibitors for Malaria. Journal of Physical Chemistry \& Biophysics. 2016;6:213. 